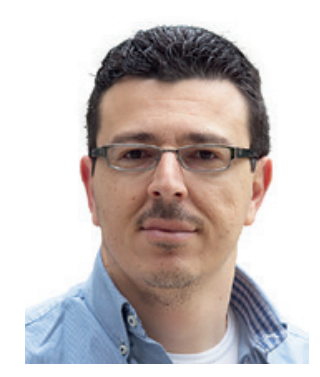

\title{
Effects of COVID-19 Home Confinement on Eating Behaviour and Physical Activity: Results of the ECLB-COVID19 International Online Survey
}

\author{
Nikolaos Papaspanos \\ Thessaloniki, Greece
}

Abstract from Ammar A, Brach M, Trabelsi K, et al.: Effects of COVID-19 home confinement on eating behaviour and physical activity: results of the ECLB-COVID19 international online survey. Nutrients. 2020;12(6):1583.

\author{
Keywords \\ COVID-19 · Nutrition · Pandemic · Physical activity · Public health
}

\begin{abstract}
Background: Public health recommendations and governmental measures during the COVID-19 pandemic have resulted in numerous restrictions on daily living including social distancing, isolation and home confinement. While these measures are imperative to abate the spreading of COVID-19, the impact of these restrictions on health behaviours and lifestyles at home is undefined. Therefore, an international online survey was launched in April 2020, in seven languages, to elucidate the behavioural and lifestyle consequences of COVID-19 restrictions. This report presents the results from the first thousand responders on physical activity (PA) and nutrition behaviours.
\end{abstract}

Subjects and methods: Following a structured review of the literature, the «Effects of home Confinement on multiple Lifestyle Behaviours during the COVID-19 outbreak (ECLB-COVID19)» Electronic survey was designed by a steering group of multidisciplinary scientists and academics. The survey was uploaded and shared on the Google online survey platform. Thirty-five research organisations from Europe, North Africa, Western Asia and the
Americas promoted the survey in English, German, French, Arabic, Spanish, Portuguese and Slovenian languages. Questions were presented in a differential format, with questions related to responses «before» and «during» confinement conditions.

Results: 1047 replies (54\% women) from Asia (36\%), Africa (40\%), Europe (21\%) and other (3\%) were included in the analysis. The COVID-19 home confinement had a negative effect on all PA intensity levels (vigorous, moderate, walking and overall). Additionally, daily sitting time increased from 5 to $8 \mathrm{~h}$ per day. Food consumption and meal patterns (the type of food, eating out of control, snacks between meals, number of main meals) were more unhealthy during confinement, with only alcohol binge drinking decreasing significantly.

Summary: While isolation is a necessary measure to protect public health, results indicate that it alters physical activity and eating behaviours in a health compromising direction. A more detailed analysis of survey data will allow for a segregation of these responses in different age groups, countries and other subgroups, which will help develop interventions to mitigate the negative lifestyle behaviours that have manifested during the COVID-19 confinement.

(c) 2020 The Authors 


\section{Knowledge Transfer}

\section{Introduction}

During the COVID-19 pandemic, there were a lot of restrictions in order to promote public health such as social distancing, isolation and home confinement. The purpose of the survey was to examine the effects of home confinement on physical activity (PA) and eating behaviour.

\section{Methods}

It was an online survey. The questionnaire was translated in different languages and included 64 questions on health, mental wellbeing, mood, life satisfaction and multidimension lifestyle behaviours (PA, diet, sleep, etc.). The questions had 2 parts: «before» and «during» home confinement. There were 1047 participants aged 18 years or older.

\section{Results}

The results show that PA was significantly decreased during home confinement compared to before. PA was decreased by $24 \%$ (days per week) and 33.5\% (minutes per day), respectively. The metabolic equivalent (MET) values for all PA decreased by $38 \%$. Additionally, the number of hours per day of sitting increased by $28.6 \%$. As for the intensity of the PA,

- the days per week with vigorous PA decreased by $22.7 \%$, the minutes per day by $33.1 \%$, and the MET values for a vigorous intensity PA by $36.9 \%$.
- the days per week for moderate PA decreased by 35\%, the minutes per day for walking by $34 \%$, and the MET values for walking by $42.7 \%$.

There were also changes to eating behaviour. During the home confinement, there was a significant increase in consuming unhealthy food, eating out of control, snacking between meals (including late-night snacks) and in the number of main meals. On the other hand, there was a decrease in alcohol binge drinking (Fig. 1).

\section{Positive Energy Balance}

The survey proves that during home confinement, the energy expenditure via PA decreased. On the other hand, the energy consumption rose by increased snacking, a higher number of meals and by choosing less healthy foods. This causes a disturbance in the energy balance as a positive energy balance is created. The higher the food consumption the higher the positive energy balance will be, something which might be triggered by anxiety and boredom evoked by quarantine. This results in excess accumulation of fat and ultimately, overweight and obese people. It is proven that obesity increases mortality in COVID-19 patients, and it is associated with many other diseases such as cardiovascular disease, diabetes, cancer, etc.

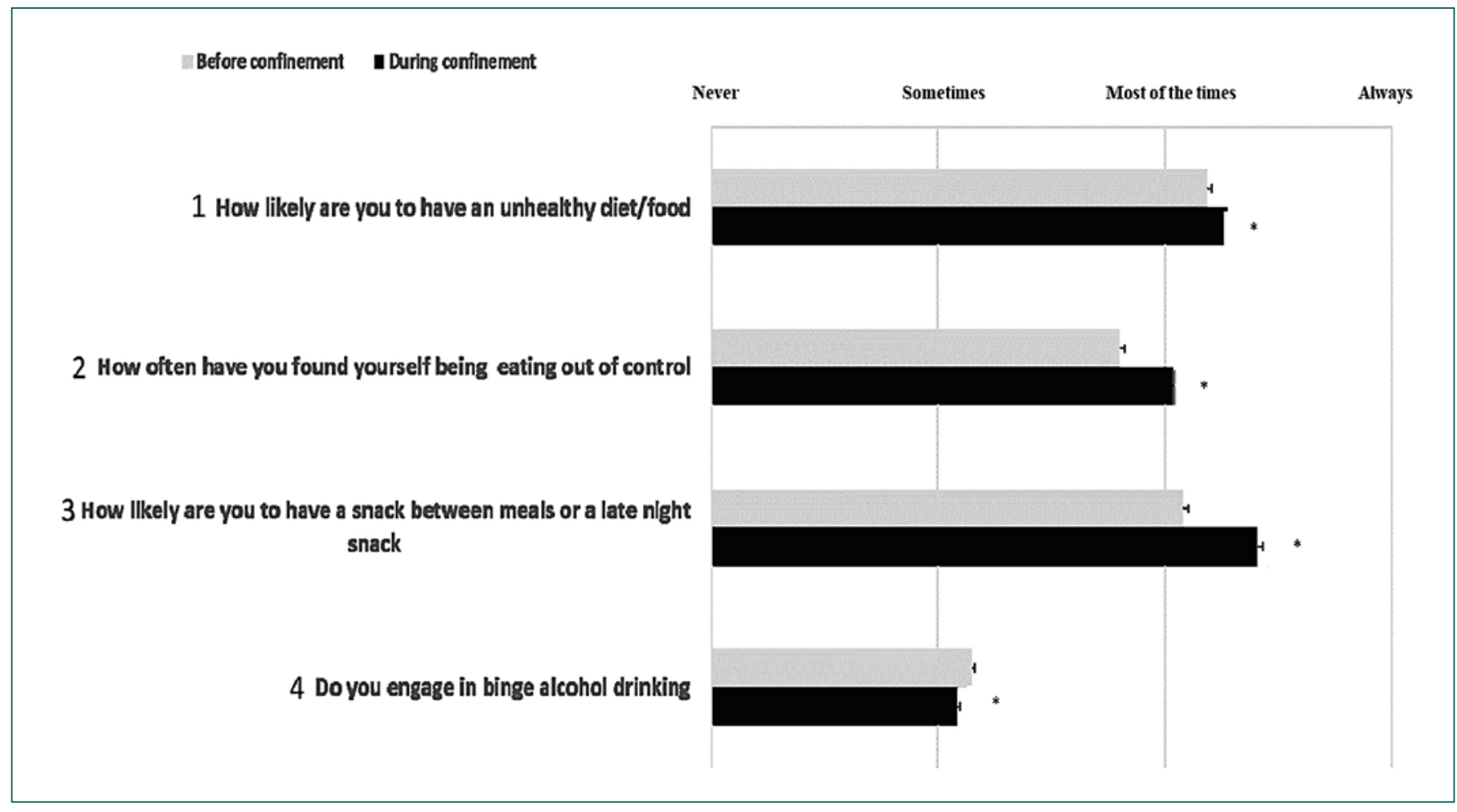

Fig. 1. Participants' scores in response to the related diet behaviour questions. * = Significant differences between "before" and "during" COVID-19 home confinement period [1]. 


\section{Lack of PA -Consequences}

The World Health Organisation (WHO) recommends for adults from

18 to 64 years old:

- At least 150 minutes of moderate-intensity PA throughout the week, or at least 75 minutes of vigorous-intensity PA throughout the week, or an equivalent combination of moderate- and vigorous-intensity activity.

- For additional health benefits, adults should increase their moderate-intensity PA to 300 minutes per week, or equivalent.

- Muscle-strengthening activities should be done involving major muscle groups on 2 or more days a week.

The survey revealed the following levels of PA during home confinement:

- vigorous PA: 26 minutes per day and 1.52 days per week (mean),

- moderate PA: 21.4 minutes per day and 1.36 days per week (mean).

There clearly is a lack of PA during home confinement. According to the $\mathrm{WHO}$, insufficient PA is one of the leading risk factors for death worldwide as well as a key risk factor for noncommunicable diseases (NCDs) such as cardiovascular diseases, cancer and diabetes. Beyond that, PA has significant health benefits and contributes to prevent NCDs.

\section{Conclusion}

Social Services and Public Health Organizations should encourage people to increase PA and obtain better food-related behaviour. As the use of technology devices, gaming platforms and social media increased during home confinement, a clever way to promote the healthy way of living could be the use of apps, games that require movement, videos on social media and, of course, role models.

\section{Disclosure Statement}

I hereby declare that there are no conflicts of interest with regard to this commentary.

\section{References}

1 Ammar A, Brach M, Trabelsi K, et al.: Effects of COVID-19 home confinement on eating behaviour and physical activity: results of the ECLB-COVID19 international online survey. Nutrients. 2020;12(6):1583.

Correspondence: Nikolaos Papaspanos, Mitropoleos 77st, 54622 Thessaloniki, Greece, nmpapaspanos@yahoo.gr 